\title{
A GENERAL VISCOSITY APPROXIMATION METHOD OF FIXED POINT SOLUTIONS OF VARIATIONAL INEQUALITIES FOR NONEXPANSIVE SEMIGROUPS IN HILBERT SPACES
}

\author{
Somyot Plubtieng and Rattanaporn Wangkeeree
}

\begin{abstract}
Let $H$ be a real Hilbert space and $\mathcal{S}=\{T(s): 0 \leq s<\infty\}$ be a nonexpansive semigroup on $H$ such that $F(\mathcal{S}) \neq \emptyset$. For a contraction $f$ with coefficient $0<\alpha<1$, a strongly positive bounded linear operator $A$ with coefficient $\bar{\gamma}>0$. Let $0<\gamma<\frac{\bar{\gamma}}{\alpha}$. It is proved that the sequences $\left\{x_{t}\right\}$ and $\left\{x_{n}\right\}$ generated by the iterative method

$$
x_{t}=t \gamma f\left(x_{t}\right)+(I-t A) \frac{1}{\lambda_{t}} \int_{0}^{\lambda_{t}} T(s) x_{t} d s
$$

and

$$
x_{n+1}=\alpha_{n} \gamma f\left(x_{n}\right)+\left(I-\alpha_{n} A\right) \frac{1}{t_{n}} \int_{0}^{t_{n}} T(s) x_{n} d s,
$$

where $\{t\},\left\{\alpha_{n}\right\} \subset(0,1)$ and $\left\{\lambda_{t}\right\},\left\{t_{n}\right\}$ are positive real divergent sequences, converges strongly to a common fixed point $\tilde{x} \in F(\mathcal{S})$ which solves the variational inequality $\langle(\gamma f-A) \tilde{x}, x-\tilde{x}\rangle \leq 0$ for $x \in F(\mathcal{S})$.
\end{abstract}

\section{Introduction}

Let $H$ be a real Hilbert space, and let $C$ be a nonempty closed convex subset of $H$. A mapping $T$ of $C$ into itself is said to be nonexpansive if $\|T x-T y\| \leq$ $\|x-y\|$ for each $x, y \in C$. We denote $F(T)$ the set of fixed points of $T$. A family $\mathcal{S}=\{T(s): 0 \leq s<\infty\}$ of mapping of $C$ into itself is called a nonexpansive semigroup on $C$ if it satisfies the following conditions:

(i) $T(0) x=x$ for all $x \in C$;

(ii) $T(s+t)=T(s) T(t)$ for all $s, t \geq 0$;

(iii) $\|T(s) x-T(s) y\| \leq\|x-y\|$ for all $x, y \in C$ and $s \geq 0$;

(iv) for all $x \in C, s \mapsto T(s) x$ is continuous.

We denote by $F(\mathcal{S})$ the set of all common fixed points of $\mathcal{S}$, that is, $F(\mathcal{S})=$ $\{x \in C: T(s) x=x$ for all $s \in S\}$. It is known that $F(\mathcal{S})$ is closed and convex.

Received February 14, 2008; Revised September 3, 2008.

2000 Mathematics Subject Classification. 46C05, 47D03, 47H09, 47H10, 47H20.

Key words and phrases. fixed point, variational inequality, viscosity approximation, nonexpansive semigroup, strong convergence.

Supported by The Commission on Higher Education. 
Construction of fixed points of nonexpansive mappings (and of common fixed points of nonexpansive semigroups) is an important subject in the theory of nonexpansive mappings and finds application in number of applied areas, in particular, in the minimization problem (see, e.g. $[4,14,15,16,17]$ ) and the references therein. A typical problem is to minimize a quadratic function over the set of the fixed points of a nonexpansive mapping on a real Hilbert space $H$ :

$$
\min _{x \in C} \frac{1}{2}\langle A x, x\rangle-\langle x, b\rangle,
$$

where $C$ is the fixed point set of a nonexpansive mapping $T$ on $H$ and $b$ is a given point in $H$.

In 2003, $\mathrm{Xu}([15])$ proved that the sequence $\left\{x_{n}\right\}$ defined by the iterative method below, with the initial guess $x_{0} \in H$ chosen arbitrarily:

$$
x_{n+1}=\left(I-\alpha_{n} A\right) T x_{n}+\alpha_{n} b, n \geq 0,
$$

converges strongly to the unique solution of the minimization problem (1.1) provided the sequence $\left\{\alpha_{n}\right\}$ satisfies certain conditions that will be made precise in Section 3.

In [6], Moudafi introduced the viscosity approximation method for nonexpansive mappings (see [13] for further developments in both Hilbert and Banach spaces). Let $f$ be a contraction on $H$. Starting with an arbitrary initial $x_{0} \in H$, define a sequence $\left\{x_{n}\right\}$ recursively by

$$
x_{n+1}=\left(1-\sigma_{n}\right) T x_{n}+\sigma_{n} f\left(x_{n}\right), n \geq 0,
$$

where $\left\{\sigma_{n}\right\}$ is a sequence in $(0,1)$. It is proved $[6,13]$ that under certain appropriate conditions imposed on $\left\{\sigma_{n}\right\}$, the sequence $\left\{x_{n}\right\}$ generated by (1.3) strongly converges to the unique solution $x^{*}$ in $C$ of the variational inequality

$$
\left\langle(I-f) x^{*}, x-x^{*}\right\rangle \geq 0, x \in C .
$$

Recently, Marino and $\mathrm{Xu}[5]$ proved the sequence $\left\{x_{n}\right\}$ generated by the iterative method $x_{n+1}=\left(I-\alpha_{n} A\right) T x_{n}+\alpha_{n} \gamma f\left(x_{n}\right)$, converges strongly to a fixed point $\tilde{x} \in F(T)$ which solves the variational inequality

$$
\left\langle(A-\gamma f) x^{*}, x-x^{*}\right\rangle \geq 0 \text { for } x \in F(T) .
$$

On the other hand, Browder [3] showed the following convergence theorem for a nonexpansive mapping: Let $T$ be a nonexpansive mapping from $C$ into itself such that the set $F(T)$ is nonempty. For each $t$ with $0<t<1$, let $u \in C$ and $x_{t}$ be an element of $C$ satisfying

$$
x_{t}=t u+(1-t) T x_{t} .
$$

Then $\left\{x_{t}\right\}$ converges strongly to the element of $F(T)$ which is nearest to $x$ in $F(T)$ as $t \downarrow 0$. This result was extended to a Banach space by Reich [8] and Takahashi and Ueda [12]. Baillon [1] proved the first nonlinear ergodic 
theorem: If $T$ is a nonexpansive mapping from $C$ into itself such that the set $F(T)$ is nonempty and $x \in C$, the Cesâro mean

$$
S_{n} x=\frac{1}{n} \sum_{k=0}^{n-1} T^{k} x
$$

converges weakly to a fixed point of $T$. Later, Baillon and Brezis [2] prove that if $\mathcal{S}=\{T(s): 0 \leq s<\infty\}$ is a nonexpansive semigroup on $C$, then the continuous scheme

$$
x_{t}=\frac{1}{t} \int_{0}^{t} T(s) x_{t} d s, \quad t \in(0,1),
$$

converges weakly to a common fixed point of $\mathcal{S}$. Those results have been generalized by several authors; see, for instance Takahashi [11]. Very recently, Plubtieng and Punpaeng [7] considered the iteration process $\left\{x_{n}\right\}$, where $x_{0} \in$ $C$ is arbitrary and

$$
x_{n+1}=\alpha_{n} f\left(x_{n}\right)+\beta_{n} x_{n}+\left(1-\alpha_{n}-\beta_{n}\right) \frac{1}{t_{n}} \int_{0}^{t_{n}} T(s) x_{n} d s
$$

for $n \geq 0$, where $\left\{\alpha_{n}\right\},\left\{\beta_{n}\right\} \subset(0,1)$ with $\alpha_{n}+\beta_{n}<1$ and $\left\{t_{n}\right\}$ is a positive real divergent sequence. They proved, under certain appropriate conditions on $\left\{\alpha_{n}\right\}$, that $\left\{x_{n}\right\}$ converges strongly to a common fixed point of $\mathcal{S}$.

The purpose of this paper is, by using the viscosity method for nonexpansive semigroups $\mathcal{S}=\{T(s): 0<s<\infty\}$ on $H$, to study the continuous scheme $\left\{x_{t}\right\}$ and the iterative scheme $\left\{x_{n}\right\}$ defined as follows: Let $T: H \rightarrow H$ be a nonexpansive mapping. For a given contraction $f$ with coefficient $0<\alpha<1$, a strongly positive bounded linear operator $A$ with coefficient $\bar{\gamma}>0,0<\gamma<\frac{\bar{\gamma}}{\alpha}$ and $t \in(0,1)$, we define a contraction mapping $T_{t}: H \rightarrow H$ by

$$
T_{t} x=t \gamma f(x)+(I-t A) \frac{1}{\lambda_{t}} \int_{0}^{\lambda_{t}} T(s) x d s, \forall x \in H,
$$

where $\left\{\lambda_{t}\right\}$ is a positive real divergent net. By Banach's contraction principle it yields a unique fixed point $x_{t} \in H$ of $T_{t}$, i.e., $x_{t}$ is the unique solution of the fixed point equation

$$
x_{t}=t \gamma f\left(x_{t}\right)+(I-t A) \frac{1}{\lambda_{t}} \int_{0}^{\lambda_{t}} T(s) x_{t} d s .
$$

A special case of (1.10) has been considered by Shioji and Takahashi [10] as follows:

$$
x_{n}=\alpha_{n} u+\left(I-\alpha_{n} A\right) \frac{1}{t_{n}} \int_{0}^{t_{n}} T(s) x_{n} d s \text { for } n \in \mathbb{N},
$$

where $\left\{\alpha_{n}\right\} \subset(0,1)$ and $\left\{t_{n}\right\}$ is a positive real divergent sequence.

Further, we also study an iteration process $\left\{x_{n}\right\}$ defined by

$$
x_{n+1}=\alpha_{n} \gamma f\left(x_{n}\right)+\left(I-\alpha_{n} A\right) \frac{1}{t_{n}} \int_{0}^{t_{n}} T(s) x_{n} d s, n \geq 0,
$$


where $x_{0} \in H,\left\{\alpha_{n}\right\}$ is a sequence in $(0,1)$ and $\left\{t_{n}\right\}$ is a positive real divergent sequence.

A special case of (1.12) was considered by Shimizu and Takahashi [9] who introduced following iterative process:

$$
x_{n+1}=\alpha_{n} u+\left(1-\alpha_{n}\right) \frac{1}{t_{n}} \int_{0}^{t_{n}} T(s) x_{n} d s, n \geq 0,
$$

where $u, x_{0} \in H$ are arbitrary (but fixed), $\left\{\alpha_{n}\right\} \subset(0,1)$ and $\left\{t_{n}\right\}$ is a positive real divergent sequence.

The purpose of this paper is to prove the strong convergence of the continuous scheme $\left\{x_{t}\right\}$ defined by (1.10) and the iterative scheme $\left\{x_{n}\right\}$ defined by (1.12) in a real Hilbert space. The results presented in this paper extend and improve the corresponding ones announced by Shioji and Takahashi [10] and Shimizu and Takahashi [9], and others.

\section{Preliminaries}

Let $H$ be a real Hilbert space with norm $\|\cdot\|$ and inner product $\langle\cdot, \cdot\rangle$ and let $C$ be a closed convex subset of $H$. Recall the metric (nearest point) projection $P_{C}$ from a Hilbert space $H$ to a closed convex subset $C$ of $H$ is defined as follows: Given $x \in H, P_{C} x$ is the only point in $C$ with the property

$$
\left\|x-P_{C} x\right\|=\inf \{\|x-y\|: y \in C\} .
$$

$P_{C} x$ is characterized as follows.

Throughout the rest of this paper, we always assume that $A$ is strongly positive; that is, there is a constant $\bar{\gamma}>0$ such that

$$
\langle A x, x\rangle \geq \bar{\gamma}\|x\|^{2}, \quad x \in H .
$$

(Note: $\bar{\gamma}>0$ is throughout reserved to be the constant such that (2.1) holds.) Recall also that a contraction on $H$ is a self-mapping $f$ of $H$ such that

$$
\|f x-f y\| \leq \alpha\|x-y\|, x, y \in H,
$$

where $\alpha \in[0,1)$ is a constant.

We need some facts and tools in a Hilbert space $H$ which are listed as lemmas below.

Lemma 2.1. Let $H$ be a real Hilbert space, $C$ a closed convex subset of $H$. Given $x \in H$ and $y \in C$. Then $y=P_{C} x$ if and only if there holds the inequality

$$
\langle x-y, y-z\rangle \geq 0, \forall z \in C \text {. }
$$

Lemma 2.2. Let $H$ be a Hilbert space, $C$ a closed convex subset of $H$, and $T: C \rightarrow C$ a nonexpansive mapping with $F(T) \neq \emptyset$. If $\left\{x_{n}\right\}$ is a sequence in $C$ weakly converging to $x \in C$ and if $\left\{(I-T) x_{n}\right\}$ converges strongly to $y$. Then $(I-T) x=y$. 
Lemma 2.3 ([13]). Assume $\left\{a_{n}\right\}$ is a sequence of nonnegative real numbers such that

$$
a_{n+1} \leq\left(1-\alpha_{n}\right) a_{n}+\delta_{n}, n \geq 0
$$

where $\left\{\alpha_{n}\right\}$ is a sequence in $(0,1)$ and $\left\{\delta_{n}\right\}$ is a sequence in $\mathbb{R}$ such that

(1) $\lim _{n \rightarrow \infty} \alpha_{n}=0$ and $\sum_{n=1}^{\infty} \alpha_{n}=\infty$,

(2) $\lim \sup _{n \rightarrow \infty} \frac{\delta_{n}}{\alpha_{n}} \leq 0$ or $\sum_{n=1}^{\infty}\left|\delta_{n}\right|<\infty$.

Then $\lim _{n \rightarrow \infty} a_{n}=0$.

Lemma 2.4 ([13]). Let $H$ be a Hilbert space, $C$ a nonempty closed convex subset of $H$, and $f: C \rightarrow C$ a contraction with coefficient $\alpha<1$. Then

$$
\langle x-y,(A-\gamma f) x-(A-\gamma f) y\rangle \geq(\bar{\gamma}-\gamma \alpha)\|x-y\|^{2}, x, y \in C .
$$

Lemma 2.5 ([5]). Assume $A$ is a strongly positive linear bounded operator on a Hilbert space $H$ with coefficient $\bar{\gamma}>0$ and $0<\rho \leq\|A\|^{-1}$. Then $\|I-\rho A\| \leq$ $1-\rho \bar{\gamma}$.

Lemma 2.6 ([9]). Let $C$ be a nonempty bounded closed convex subset of $H$ and let $\mathcal{S}=\{T(s): 0 \leq s<\infty\}$ be a nonexpansive semigroup on $C$. Then, for any $h \geq 0$,

$$
\lim _{t \rightarrow \infty} \sup _{x \in C}\left\|\frac{1}{t} \int_{0}^{t} T(s) x d s-T(h)\left(\frac{1}{t} \int_{0}^{t} T(s) x d s\right)\right\|=0 .
$$

\section{Main results}

In this section, we prove two strong convergence theorems of the continuous scheme $\left\{x_{t}\right\}$ defined by (1.10) and the iterative scheme $\left\{x_{n}\right\}$ defined by (1.12).

Theorem 3.1. Let $H$ be a real Hilbert space $H$ and $\mathcal{S}=\{T(s): 0 \leq s<\infty\}$ be a nonexpansive semigroup on $H$ such that $F(\mathcal{S}) \neq \emptyset$. Let $A$ be a bounded linear operator on $H$, and let $t \in(0,1)$ such that $t \leq\|A\|^{-1}$ and $0<\gamma<\frac{\bar{\gamma}}{\alpha}$ which satisfies $t \rightarrow 0$. Then for a contraction mapping $f: H \rightarrow H$ with coefficient $\alpha \in(0,1)$, the sequence $\left\{x_{t}\right\}$ defined by (1.10) with $\lambda_{t}$ is a positive real divergent sequence, converges strongly to $\tilde{x}, \tilde{x}$ is the unique solution in $F(\mathcal{S})$ of the variational inequality

$$
\langle(A-\gamma f) \tilde{x}, x-\tilde{x}\rangle \geq 0, x \in F(\mathcal{S})
$$

or equivalently $\tilde{x}=P_{F(\mathcal{S})}(I-A+\gamma f) \tilde{x}$, where $P$ is a metric projection mapping from $H$ onto $F(\mathcal{S})$.

Proof. Observe that for $t \in\left(0,\|A\|^{-1}\right)$, we have $\|I-t A\| \leq 1-t \bar{\gamma}$ by Lemma 2.5 . Let $q \in F(S)$. We note that

$$
\begin{aligned}
\left\|x_{t}-q\right\| & =\left\|t \gamma f\left(x_{t}\right)+(I-t A) \frac{1}{\lambda_{t}} \int_{0}^{\lambda_{t}} T(s) x_{t} d s-q\right\| \\
& \leq t\left\|\gamma f\left(x_{t}\right)-A q\right\|+\|I-t A\| \frac{1}{\lambda_{t}} \int_{0}^{\lambda_{t}}\left\|T(s) x_{t}-q\right\| d s
\end{aligned}
$$




$$
\begin{aligned}
& \leq t\left\|\gamma f\left(x_{t}\right)-A q\right\|+(1-t \bar{\gamma})\left\|x_{t}-q\right\| \\
& \leq t\left\|\gamma\left(f\left(x_{t}\right)-f(q)\right)+(\gamma f(q)-A q)\right\|+(1-t \bar{\gamma})\left\|x_{t}-q\right\| \\
& \leq t\left(\gamma \alpha\left\|x_{t}-q\right\|+\|\gamma f(q)-A q\|\right)+(1-t \bar{\gamma})\left\|x_{t}-q\right\| \\
& =(1-t(\bar{\gamma}-\gamma \alpha))\left\|x_{t}-q\right\|+t\|\gamma f(q)-A q\| .
\end{aligned}
$$

It follows that

$$
\left\|x_{t}-q\right\| \leq \frac{\|\gamma f(q)-A q\|}{\bar{\gamma}-\gamma \alpha}
$$

Hence $\left\{x_{t}\right\}$ is bounded, and so is $\left\{f\left(x_{t}\right)\right\}$. Further, we note that

$$
\begin{aligned}
\left\|T(h) x_{t}-x_{t}\right\|= & \left\|T(h) x_{t}-T(h)\left(\frac{1}{\lambda_{t}} \int_{0}^{\lambda_{t}} T(s) x_{t} d s\right)\right\| \\
& +\left\|T(h)\left(\frac{1}{\lambda_{t}} \int_{0}^{\lambda_{t}} T(s) x_{t} d s\right)-\frac{1}{\lambda_{t}} \int_{0}^{\lambda_{t}} T(s) x_{t} d s\right\| \\
& +\left\|\frac{1}{\lambda_{t}} \int_{0}^{\lambda_{t}} T(s) x_{t} d s-x_{t}\right\| \\
\leq & 2\left\|\frac{1}{\lambda_{t}} \int_{0}^{\lambda_{t}} T(s) x_{t} d s-x_{t}\right\| \\
& +\left\|T(h)\left(\frac{1}{\lambda_{t}} \int_{0}^{\lambda_{t}} T(s) x_{t} d s\right)-\frac{1}{\lambda_{t}} \int_{0}^{\lambda_{t}} T(s) x_{t} d s\right\|
\end{aligned}
$$

for every $0 \leq h<\infty$. On the other hand, we note that

$$
\left\|\frac{1}{\lambda_{t}} \int_{0}^{\lambda_{t}} T(s) x_{t} d s-x_{t}\right\|=t\left\|A\left(\frac{1}{\lambda_{t}} \int_{0}^{\lambda_{t}} T(s) x_{t} d s\right)-\gamma f\left(x_{t}\right)\right\|
$$

for every $t>0$. Let $z_{0} \in F(\mathcal{S})$ and $D=\left\{z \in H:\left\|z-z_{0}\right\| \leq \frac{1}{\bar{\gamma}-\gamma \alpha} \| \gamma f\left(z_{0}\right)-\right.$ $\left.A z_{0} \|\right\}$. Then, $D$ is a nonempty closed bounded convex subset of $C$ which is $T(s)$-invariant for each $s \in[0, \infty)$ and contains $\left\{x_{t}\right\}$, it follows by Lemma 2.6 that

$$
\lim _{\lambda_{t} \rightarrow \infty}\left\|T(h)\left(\frac{1}{\lambda_{t}} \int_{0}^{\lambda_{t}} T(s) x_{t} d s\right)-\frac{1}{\lambda_{t}} \int_{0}^{\lambda_{t}} T(s) x_{t} d s\right\|=0
$$

for every $0 \leq h<\infty$. By (3.2)-(3.4) and $t \rightarrow 0$, we obtain

$$
\left\|T(h) x_{t}-x_{t}\right\| \rightarrow 0
$$

for each $0 \leq h<\infty$. Assume $\left\{t_{n}\right\}_{n=1}^{\infty} \subset(0,1)$ is such that $t_{n} \rightarrow 0$ as $n \rightarrow \infty$. Put $x_{n}:=x_{t_{n}}$ and $\lambda_{n}:=\lambda_{t_{n}}$. We will show that $\left\{x_{n}\right\}$ contains a subsequence converging strongly to $\tilde{x}$, where $\tilde{x} \in F(\mathcal{S})$ is the unique solution of the variational inequality (3.1). Since $\left\{x_{n}\right\}$ is a bounded sequence, there is a subsequence $\left\{x_{n_{j}}\right\}$ of $\left\{x_{n}\right\}$ which converges weakly to $\tilde{x} \in H$. By Lemma 2.2, 
we have $\tilde{x} \in F(\mathcal{S})$. For each $n \geq 1$, we note that

$$
x_{n}-\tilde{x}=t_{n}\left(\gamma f\left(x_{n}\right)-A \tilde{x}\right)+\left(I-t_{n} A\right)\left(\frac{1}{\lambda_{n}} \int_{0}^{\lambda_{n}} T(s) x_{n} d s-\tilde{x}\right) .
$$

Thus, we have

$$
\begin{aligned}
& \left\|x_{n}-\tilde{x}\right\|^{2} \\
= & t_{n}\left\langle\gamma f\left(x_{n}\right)-A \tilde{x}, x_{n}-\tilde{x}\right\rangle+\left\langle\left(I-t_{n} A\right)\left(\frac{1}{\lambda_{n}} \int_{0}^{\lambda_{n}} T(s) x_{n} d s-\tilde{x}\right), x_{n}-\tilde{x}\right\rangle \\
\leq & t_{n}\left\langle\gamma f\left(x_{n}\right)-A \tilde{x}, x_{n}-\tilde{x}\right\rangle+\left\|I-t_{n} A\right\|\left\|\frac{1}{\lambda_{n}} \int_{0}^{\lambda_{n}}\left(T(s) x_{n}-\tilde{x}\right) d s\right\|\left\|x_{n}-\tilde{x}\right\| \\
\leq & t_{n}\left\langle\gamma f\left(x_{n}\right)-A \tilde{x}, x_{n}-\tilde{x}\right\rangle+\left(1-t_{n} \bar{\gamma}\right)\left\|x_{n}-\tilde{x}\right\|\left(\frac{1}{\lambda_{n}} \int_{0}^{\lambda_{n}}\left\|T(s) x_{n}-\tilde{x}\right\| d s\right) \\
\leq & t_{n}\left\langle\gamma f\left(x_{n}\right)-A \tilde{x}, x_{n}-\tilde{x}\right\rangle+\left(1-t_{n} \bar{\gamma}\right)\left\|x_{n}-\tilde{x}\right\|\left(\frac{1}{\lambda_{n}} \int_{0}^{\lambda_{n}}\left\|x_{n}-\tilde{x}\right\| d s\right) \\
\leq & t_{n}\left\langle\gamma f\left(x_{n}\right)-A \tilde{x}, x_{n}-\tilde{x}\right\rangle+\left(1-t_{n} \bar{\gamma}\right)\left\|x_{n}-\tilde{x}\right\|^{2} .
\end{aligned}
$$

Hence

$$
\begin{aligned}
\left\|x_{n}-\tilde{x}\right\|^{2} & \leq \frac{1}{\bar{\gamma}}\left\langle\gamma f\left(x_{n}\right)-A \tilde{x}, x_{n}-\tilde{x}\right\rangle \\
& =\frac{1}{\bar{\gamma}}\left\{\gamma\left\langle f\left(x_{n}\right)-f(\tilde{x}), x_{n}-\tilde{x}\right\rangle+\left\langle\gamma f(\tilde{x})-A \tilde{x}, x_{n}-\tilde{x}\right\rangle\right\} \\
& \leq \frac{1}{\bar{\gamma}}\left\{\gamma \alpha\left\|x_{n}-\tilde{x}\right\|^{2}+\left\langle\gamma f(\tilde{x})-A \tilde{x}, x_{n}-\tilde{x}\right\rangle\right\} .
\end{aligned}
$$

This implies that

$$
\left\|x_{n}-\tilde{x}\right\|^{2} \leq \frac{1}{\bar{\gamma}-\gamma \alpha}\left\langle\gamma f(\tilde{x})-A \tilde{x}, x_{n}-\tilde{x}\right\rangle .
$$

In particular, we have

$$
\left\|x_{n_{j}}-\tilde{x}\right\|^{2} \leq \frac{1}{\bar{\gamma}-\gamma \alpha}\left\langle\gamma f(\tilde{x})-A \tilde{x}, x_{n_{j}}-\tilde{x}\right\rangle .
$$

Since $x_{n_{j}} \rightarrow \tilde{x}$, it follows from (3.5) that $x_{n_{j}} \rightarrow \tilde{x}$ as $j \rightarrow \infty$. Next, we show that $\tilde{x} \in F(\mathcal{S})$ solves the variational inequality (3.1). Indeed, we note that $x_{t}$ solves the fixed point equation

$$
x_{t}=t \gamma f\left(x_{t}\right)+(I-t A) \frac{1}{\lambda_{t}} \int_{0}^{\lambda_{t}} T(s) x_{t} d s .
$$

Thus, we have

$$
(A-\gamma f) x_{t}=-\frac{1}{t}(I-t A)\left(x_{t}-\frac{1}{\lambda_{t}} \int_{0}^{\lambda_{t}} T(s) x_{t} d s\right) .
$$


Since $I-T$ is monotone (i.e., $\langle x-y,(I-T) x-(I-T) y\rangle \geq 0$ for $x, y \in H)$, it follows that, for each $q \in F(\mathcal{S})$ and for all $t>0$,

$$
\begin{aligned}
& \left\langle(A-\gamma f) x_{t}, x_{t}-q\right\rangle \\
= & -\frac{1}{t}\left\langle(I-t A)\left(x_{t}-\frac{1}{\lambda_{t}} \int_{0}^{\lambda_{t}} T(s) x_{t} d s\right), x_{t}-q\right\rangle \\
= & -\frac{1}{t}\left\langle(I-t A)\left(\frac{1}{\lambda_{t}} \int_{0}^{\lambda_{t}} x_{t} d s-\frac{1}{\lambda_{t}} \int_{0}^{\lambda_{t}} T(s) x_{t} d s\right), x_{t}-q\right\rangle \\
= & -\frac{1}{t}\left\langle\frac{1}{\lambda_{t}} \int_{0}^{\lambda_{t}}(I-T(s)) x_{t} d s-\frac{1}{\lambda_{t}} \int_{0}^{\lambda_{t}}(I-T(s)) q d s, x_{t}-q\right\rangle \\
& +\left\langle A\left(\frac{1}{\lambda_{t}} \int_{0}^{\lambda_{t}}(I-T(s)) x_{t} d s\right), x_{t}-q\right\rangle \\
= & -\left(\frac{1}{t}\right) \frac{1}{\lambda_{t}} \int_{0}^{\lambda_{t}}\left\langle(I-T(s)) x_{t}-(I-T(s)) q, x_{t}-q\right\rangle d s \\
& +\left\langle A\left(\frac{1}{\lambda_{t}} \int_{0}^{\lambda_{t}}(I-T(s)) x_{t} d s\right), x_{t}-q\right\rangle \\
\leq & \left\langle A\left(\frac{1}{\lambda_{t}} \int_{0}^{\lambda_{t}}(I-T(s)) x_{t} d s\right), x_{t}-q\right\rangle .
\end{aligned}
$$

Now replacing $t$ and $\lambda_{t}$ in (3.6) with $t_{n_{j}}$ and $\lambda_{n_{j}}$, respectively, and letting $j \rightarrow \infty$, we noticing that $(I-T(s)) x_{n_{j}} \rightarrow(I-T(s)) \tilde{x}=0$ for $\tilde{x} \in F(\mathcal{S})$. This implies that

$$
\langle(A-\gamma f) \tilde{x}, \tilde{x}-q\rangle \leq 0, \forall q \in F(\mathcal{S}) .
$$

That is, $\tilde{x}$ is a solution of (3.1). We next to show that the net $\left\{x_{t}\right\}$ convergence strongly to $\tilde{x}$. Assume that there is a sequence $k_{n} \subset(0,1)$ such that $x_{k_{n}} \rightarrow x^{\prime}$, where $k_{n} \rightarrow 0$. We note Lemma 2.2 that $x^{\prime} \in F(\mathcal{S})$. It follows from the inequality (3.7) that

$$
\left\langle(A-\gamma f) \tilde{x}, \tilde{x}-x^{\prime}\right\rangle \leq 0 .
$$

Interchange $\tilde{x}$ and $x^{\prime}$ to obtain

$$
\left\langle(A-\gamma f) x^{\prime}, x^{\prime}-\tilde{x}\right\rangle \leq 0 .
$$

Adding (3.8) and (3.9) and by Lemma 2.4, we get

$$
(\bar{\gamma}-\gamma \alpha)\left\|\tilde{x}-x^{\prime}\right\|^{2} \leq\left\langle\tilde{x}-x^{\prime},(A-\gamma f) \tilde{x}-(A-\gamma f) x^{\prime}\right\rangle \leq 0 .
$$

By Lemma 2.4, we have $\tilde{x}=x^{\prime}$. Hence $\left\{x_{t}\right\}$ converges strongly to $\tilde{x}$.

Fix $u \in C \subseteq H$, taking $f(x)=u$ for all $x \in H, A=I$ and $\gamma=1$ in Theorem 3.1, we get the following corollary.

Corollary 3.2 (Shioji and Takahashi[10]). Let $C$ be a closed convex subset of a Hilbert space $H$. Let $\mathcal{S}=\{T(s): 0 \leq s<\infty\}$ be a strongly continuous semigroup of nonexpansive mapping on $C$ such that $F(\mathcal{S}) \neq \emptyset$. Let $\left\{\alpha_{n}\right\}$ and 
$\left\{t_{n}\right\}$ be positive sequences of real numbers satisfying $0<\alpha_{n}<1, \lim _{n \rightarrow \infty} \alpha_{n}=$ 0 and $\lim _{n \rightarrow \infty} t_{n}=\infty$. Fix $u \in C$ and define a sequence $\left\{x_{n}\right\}$ in $C$ by (1.11) for $n \in \mathbb{N}$. Then $\left\{x_{n}\right\}$ converges strongly to the element of $F(\mathcal{S})$ nearest to $u$.

Theorem 3.3. Let $H$ be a real Hilbert space and $\mathcal{S}=\{T(s): 0 \leq s<\infty\}$ be a nonexpansive semigroup on $H$ such that $F(\mathcal{S}) \neq \emptyset$. Then for a contraction mapping $f: H \rightarrow H$ with coefficient $\alpha \in(0,1)$ and $A$ is a bounded linear operator on $H$, the sequence $\left\{\alpha_{n}\right\}$ of parameters satisfying $0<\alpha_{n}<1, \lim _{n \rightarrow \infty} \alpha_{n}=$ $0, \sum_{n=0}^{\infty} \alpha_{n}=\infty$ and $\lim _{n \rightarrow \infty} t_{n}=\infty$, the sequence $\left\{x_{n}\right\}$ defined by (1.12) converges strongly to $\tilde{x}, \tilde{x}$ is the unique solution in $F(\mathcal{S})$ of the variational inequality

$$
\langle(A-\gamma f) \tilde{x}, x-\tilde{x}\rangle \geq 0, x \in F(\mathcal{S})
$$

or equivalently $\tilde{x}=P_{F(\mathcal{S})}(I-A+\gamma f) \tilde{x}$, where $P$ is a metric projection mapping from $H$ onto $F(\mathcal{S})$.

Proof. Since $\alpha_{n} \rightarrow 0$, we may assume, without loss of generality, that $\alpha_{n}<$ $\|A\|^{-1}$ for all $n$. We first show that $\left\{x_{n}\right\}$ is bounded. Let $q \in F(\mathcal{S})$, we note that

$$
\begin{aligned}
\left\|x_{n+1}-q\right\| & =\left\|\alpha_{n} \gamma f\left(x_{n}\right)+\left(I-\alpha_{n} A\right) \frac{1}{t_{n}} \int_{0}^{t_{n}} T(s) x_{n} d s-q\right\| \\
& \leq \alpha_{n}\left\|\gamma f\left(x_{n}\right)-A q\right\|+\left\|I-\alpha_{n} A\right\| \frac{1}{t_{n}} \int_{0}^{t_{n}}\left\|T(s) x_{n}-q\right\| d s \\
& \leq \alpha_{n} \gamma\left\|f\left(x_{n}\right)-f(q)\right\|+\alpha_{n}\|\gamma f(q)-A q\|+\left(1-\alpha_{n} \bar{\gamma}\right)\left\|x_{n}-q\right\| \\
& \leq \alpha_{n} \gamma \alpha\left\|x_{n}-q\right\|+\alpha_{n}\|\gamma f(q)-A q\|+\left(1-\alpha_{n} \bar{\gamma}\right)\left\|x_{n}-q\right\| \\
& =\left(1-(\bar{\gamma}-\gamma \alpha) \alpha_{n}\right)\left\|x_{n}-q\right\|+\alpha_{n}\|\gamma f(q)-A q\| \\
& =\left(1-(\bar{\gamma}-\gamma \alpha) \alpha_{n}\right)\left\|x_{n}-q\right\|+(\bar{\gamma}-\gamma \alpha) \alpha_{n} \frac{\|\gamma f(q)-A q\|}{\bar{\gamma}-\gamma \alpha}, \forall n \geq 0 .
\end{aligned}
$$

By induction, we get

$$
\left\|x_{n}-q\right\| \leq \max \left\{\left\|x_{0}-q\right\|, \frac{\|\gamma f(q)-A q\|}{\bar{\gamma}-\gamma \alpha}\right\}, n \geq 0 .
$$

This implies that $\left\{x_{n}\right\}$ and $f\left(x_{n}\right)$ are bounded. Put $z_{0}=P_{F(\mathcal{S})} x_{0}$ and set $D=\left\{z \in H:\left\|z-z_{0}\right\| \leq\left\|x_{0}-z_{0}\right\|+\frac{1}{\bar{\gamma}-\gamma \alpha}\left\|\gamma f\left(z_{0}\right)-A z_{0}\right\|\right\}$. Then $D$ is nonempty closed bounded convex subset of $H$ which $T(s)$-invariant for each $s \in[0, \infty)$ and contains $\left\{x_{n}\right\}$. So without loss of generality, we may assume $\mathcal{S}=\{T(s): 0 \leq s<\infty\}$ is nonexpansive semigroup on $D$. By Lemma 2.6, we get

$$
\lim _{n \rightarrow \infty}\left\|\frac{1}{t_{n}} \int_{0}^{t_{n}} T(s) x_{n} d s-T(h)\left(\frac{1}{t_{n}} \int_{0}^{t_{n}} T(s) x_{n} d s\right)\right\|=0
$$


for every $h \in[0, \infty)$. Let $y_{n}:=\frac{1}{t_{n}} \int_{0}^{t_{n}} T(s) x_{n} d s$ and let $\tilde{x}$ be the unique solution in $F(\mathcal{S})$ of the variational in equality (3.10). Next we show that

$$
\limsup _{n \rightarrow \infty}\left\langle\tilde{x}-y_{n}, A \tilde{x}-\gamma f(\tilde{x})\right\rangle \leq 0 .
$$

Let $\left\{y_{n_{j}}\right\}$ be a subsequence of $\left\{y_{n}\right\}$ such that

$$
\lim _{j \rightarrow \infty}\left\langle\tilde{x}-y_{n_{j}}, A \tilde{x}-\gamma f(\tilde{x})\right\rangle=\limsup _{n \rightarrow \infty}\left\langle\tilde{x}-y_{n}, A \tilde{x}-\gamma f(\tilde{x})\right\rangle,
$$

and $y_{n_{j}} \rightarrow \tilde{q} \in H$. By Opial's condition, we have $\tilde{q} \in F(\mathcal{S})$. In fact, if $\tilde{q} \neq T(h) \tilde{q}$ for some $h \in[0, \infty)$, we have

$$
\begin{aligned}
\liminf _{j \rightarrow \infty}\left\|y_{n_{j}}-\tilde{q}\right\| & <\liminf _{j \rightarrow \infty}\left\|y_{n_{j}}-T(h) \tilde{q}\right\| \\
& \leq \liminf _{j \rightarrow \infty}\left(\left\|y_{n_{j}}-T(h) y_{n_{j}}\right\|+\left\|T(h) y_{n_{j}}-T(h) \tilde{q}\right\|\right) \\
& \leq \liminf _{j \rightarrow \infty}\left\|y_{n_{j}}-\tilde{q}\right\| .
\end{aligned}
$$

This is a contradiction. Therefore, we have $\tilde{q}=T(h) \tilde{q}$ for each $h \geq 0$ and so $\tilde{q} \in F(\mathcal{S})$. Hence by $(3.10)$, we obtain

$$
\limsup _{n \rightarrow \infty}\left\langle\tilde{x}-y_{n}, A \tilde{x}-\gamma f(\tilde{x})\right\rangle=\langle\tilde{x}-\tilde{q}, A \tilde{x}-\gamma f(\tilde{x})\rangle \leq 0
$$

as required. Finally we shall show that $x_{n} \rightarrow \tilde{x}$. For each $n \geq 0$, we have

$$
\begin{aligned}
& \left\|x_{n+1}-\tilde{x}\right\|^{2} \\
= & \left\|\left(I-\alpha_{n} A\right)\left(\frac{1}{t_{n}} \int_{0}^{t_{n}} T(s) x_{n} d s-\tilde{x}\right)+\alpha_{n}\left(\gamma f\left(x_{n}\right)-A \tilde{x}\right)\right\|^{2} \\
= & \left\|\left(I-\alpha_{n} A\right) \frac{1}{t_{n}} \int_{0}^{t_{n}} T(s) x_{n} d s-\tilde{x}\right\|^{2}+\alpha_{n}^{2}\left\|\gamma f\left(x_{n}\right)-A \tilde{x}\right\|^{2} \\
& +2 \alpha_{n}\left\langle\left(I-\alpha_{n} A\right) \frac{1}{t_{n}} \int_{0}^{t_{n}} T(s) x_{n} d s-\tilde{x}, \gamma f\left(x_{n}\right)-A \tilde{x}\right\rangle \\
\leq & \left(1-\alpha_{n} \bar{\gamma}\right)^{2}\left\|x_{n}-\tilde{x}\right\|^{2}+\alpha_{n}^{2}\left\|\gamma f\left(x_{n}\right)-A \tilde{x}\right\|^{2} \\
& +2 \alpha_{n} \gamma\left\langle\frac{1}{t_{n}} \int_{0}^{t_{n}} T(s) x_{n} d s-\tilde{x}, f\left(x_{n}\right)-f(\tilde{x})\right\rangle \\
& +2 \alpha_{n}\left\langle\frac{1}{t_{n}} \int_{0}^{t_{n}} T(s) x_{n} d s-\tilde{x}, \gamma f(\tilde{x})-A \tilde{x}\right\rangle \\
& -2 \alpha_{n}^{2}\left\langle A\left(\frac{1}{t_{n}} \int_{0}^{t_{n}} T(s) x_{n} d s-\tilde{x}\right), \gamma f\left(x_{n}\right)-A \tilde{x}\right\rangle \\
\leq & {\left[\left(1-\alpha_{n} \bar{\gamma}\right)^{2}+2 \alpha_{n} \gamma \alpha\right]\left\|x_{n}-\tilde{x}\right\|^{2} } \\
& +\alpha_{n}\left[2\left\langle\frac{1}{t_{n}} \int_{0}^{t_{n}} T(s) x_{n} d s-\tilde{x}, \gamma f(\tilde{x})-A \tilde{x}\right\rangle+\alpha_{n}\left(\left\|\gamma f\left(x_{n}\right)-A \tilde{x}\right\|^{2}\right.\right. \\
& \left.\left.+2\left\|A\left(\frac{1}{t_{n}} \int_{0}^{t_{n}} T(s) x_{n} d s-\tilde{x}\right)\right\|\left\|\gamma f\left(x_{n}\right)-A(\tilde{x})\right\|\right)\right]
\end{aligned}
$$




$$
\begin{aligned}
\leq & \left(1-2(\bar{\gamma}-\gamma \alpha) \alpha_{n}\right)\left\|x_{n}-\tilde{x}\right\|^{2} \\
& +\alpha_{n}\left\{2\left\langle\frac{1}{t_{n}} \int_{0}^{t_{n}} T(s) x_{n} d s-\tilde{x}, \gamma f(\tilde{x})-A \tilde{x}\right\rangle+\alpha_{n}\left(\left\|\gamma f\left(x_{n}\right)-A \tilde{x}\right\|^{2}\right.\right. \\
& \left.\left.+2\left\|A\left(\frac{1}{t_{n}} \int_{0}^{t_{n}} T(s) x_{n} d s-\tilde{x}\right)\right\|\left\|\gamma f\left(x_{n}\right)-A(\tilde{x})\right\|+\bar{\gamma}^{2}\left\|x_{n}-\tilde{x}\right\|^{2}\right)\right\} \\
= & \left(1-2(\bar{\gamma}-\gamma \alpha) \alpha_{n}\right)\left\|x_{n}-\tilde{x}\right\|^{2}+\alpha_{n} \beta_{n}
\end{aligned}
$$

where $\beta_{n}=2\left\langle\frac{1}{t_{n}} \int_{0}^{t_{n}} T(s) x_{n} d s-\tilde{x}, \gamma f(\tilde{x})-A \tilde{x}\right\rangle+\alpha_{n} L$ and $L \geq\left\|\gamma f\left(x_{n}\right)-A \tilde{x}\right\|^{2}+$ $2\left\|A\left(\frac{1}{t_{n}} \int_{0}^{t_{n}} T(s) x_{n} d s-\tilde{x}\right)\right\|\left\|\gamma f\left(x_{n}\right)-A(\tilde{x})\right\|+\bar{\gamma}^{2}\left\|x_{n}-\tilde{x}\right\|^{2}$ for all $n \geq 0$. It is easily seen that $2(\bar{\gamma}-\gamma \alpha) \alpha_{n} \rightarrow 0, \sum_{n=1}^{\infty} 2(\bar{\gamma}-\gamma \alpha) \alpha_{n}=\infty$ and $\lim _{\sup _{n \rightarrow \infty}} \frac{\beta_{n}}{2(\bar{\gamma}-\gamma \alpha)} \leq$ 0 by (3.11). By Lemma 2.3, the sequence $\left\{x_{n}\right\}$ converges strongly to a fixed point $\tilde{x}$ of $T$.

Fix $u \in C \subseteq H$, taking $f(x)=u$ for all $x \in H, A=I$ and $\gamma=1$ in Theorem 3.3, we get the following corollary.

Corollary 3.4 (Shimizu and Takahashi [9]). Let $C$ be a nonempty closed convex subset of a real Hilbert space $H$. and $\mathcal{S}=\{T(s): 0 \leq s<\infty\}$ be a nonexpansive semigroup on $C$ such that $F(\mathcal{S}) \neq \emptyset$. Let $\left\{\alpha_{n}\right\}$ be a sequence in $(0,1)$ which satisfies $\lim _{n \rightarrow \infty} \alpha_{n}=0$ and $\sum_{n=1}^{\infty} \alpha_{n}=\infty$. Let $u \in C$ and $\left\{t_{n}\right\}$ is a positive real divergent sequence, then the sequence $\left\{x_{n}\right\}$ defined by (1.13) converges strongly to a common fixed point $P_{F(\mathcal{S})} u$.

\section{References}

[1] J. B. Baillon, Un théorème de type ergodique pour les contractions non linéaires dans un espace de Hilbert, C. R. Acad. Sci. Paris Sér. A-B 280 (1975), no. 22, Aii, A1511-A1514.

[2] J. B. Baillon and H. Brézis, Une remarque sur le comportement asymptotique des semigroupes non linéaires, Houston J. Math. 2 (1976), no. 1, 5-7.

[3] F. E. Browder, Convergence of approximants to fixed points of nonexpansive non-linear mappings in Banach spaces, Arch. Rational Mech. Anal. 24 (1967), 82-90.

[4] F. Deutsch and I. Yamada, Minimizing certain convex functions over the intersection of the fixed point sets of nonexpansive mappings, Numer. Funct. Anal. Optim. 19 (1998), no. $1-2,33-56$.

[5] G. Marino and H. K. Xu, A general iterative method for nonexpansive mappings in Hilbert spaces, J. Math. Anal. Appl. 318 (2006), no. 1, 43-52.

[6] A. Moudafi, Viscosity approximation methods for fixed-points problems, J. Math. Anal. Appl. 241 (2000), no. 1, 46-55.

[7] S. Plubtieng and R. Punpaeng, Fixed-point solutions of variational inequalities for nonexpansive semigroups in Hilbert spaces, Math. Comput. Modelling 48 (2008), no. 1-2, $279-286$.

[8] S. Reich, Strong convergence theorems for resolvents of accretive operators in Banach spaces, J. Math. Anal. Appl. 75 (1980), no. 1, 287-292.

[9] T. Shimizu and W. Takahashi, Strong convergence to common fixed points of families of nonexpansive mappings, J. Math. Anal. Appl. 211 (1997), no. 1, 71-83.

[10] N. Shioji and W. Takahashi, Strong convergence theorems for asymptotically nonexpansive semigroups in Hilbert spaces, Nonlinear Anal. 34 (1998), no. 1, 87-99. 
[11] W. Takahashi, Nonlinear Functional Analysis, Fixed point theory and its applications. Yokohama Publishers, Yokohama, 2000.

[12] W. Takahashi and Y. Ueda, On Reich's strong convergence theorems for resolvents of accretive operators, J. Math. Anal. Appl. 104 (1984), no. 2, 546-553.

[13] H. K. Xu, Viscosity approximation methods for nonexpansive mappings, J. Math. Anal. Appl. 298 (2004), no. 1, 279-291.

[14] _ Iterative algorithms for nonlinear operators, J. London Math. Soc. (2) 66 (2002), no. 1, 240-256.

[15] _ An iterative approach to quadratic optimization, J. Optim. Theory Appl. 116 (2003), no. 3, 659-678.

[16] I. Yamada, The hybrid steepest descent method for the variational inequality problem over the intersection of fixed point sets of nonexpansive mappings, Inherently parallel algorithms in feasibility and optimization and their applications (Haifa, 2000), 473-504, Stud. Comput. Math., 8, North-Holland, Amsterdam, 2001.

[17] I. Yamada, N. Ogura, Y. Yamashita, and K. Sakaniwa, Quadratic optimization of fixed points of nonexpansive mappings in Hilbert space, Numer. Funct. Anal. Optim. 19 (1998), no. 1-2, 165-190.

Somyot Plubtieng

Department of Mathematics

FaCUlty of SCience

NARESUAN UNIVERSITY

Phitsanulok 65000, Thailand

E-mail address: Somyotp@nu.ac.th

RATTANAPORN WANGKEEREE

DepaRtMent of Mathematics

FACULTY OF SCIENCE

Naresuan University

Phitsanulok 65000, Thailand 